\title{
Randomized study of teriflunomide effects on immune responses to neoantigen and recall antigens
}

\section{OPEN}

Amit Bar-Or, MD

Heinz Wiendl, MD

Barry Miller, MA

Myriam Benamor, MD

Philippe Truffinet, MD

Meg Church, MS

Francoise Menguy-

Vacheron, PhD

Correspondence to

Dr. Bar-Or:

amit.bar-or@mcgill.ca
Supplemental data at Neurology.org/nn

\section{ABSTRACT}

Objective: To evaluate immune responses to neoantigen and recall antigens in healthy subjects treated with teriflunomide.

Methods: This was a randomized, double-blind, placebo-controlled study. Subjects received oral teriflunomide (70 mg once daily for 5 days followed by $14 \mathrm{mg}$ once daily for 25 days) or placebo for 30 days. Antibody responses were evaluated following rabies vaccination (neoantigen) applied at days 5, 12, and 31 of the treatment period. Occurrence of delayed-type hypersensitivity (DTH) to Candida albicans, Trichophyton, and tuberculin (recall antigens) was assessed before and at the end of treatment to investigate cellular memory response. Safety and pharmacokinetics were evaluated.

Results: Forty-six randomized subjects were treated (teriflunomide, $\mathrm{n}=23$; placebo, $\mathrm{n}=23$ ) and completed the rabies vaccination. Geometric mean titers for rabies antibodies were lower with teriflunomide at days 31 and 38 than with placebo. However, all subjects achieved sufficient seroprotection following rabies vaccination (titers well above the $0.5 \mathrm{lU} / \mathrm{mL}$ threshold). Overall, the DTH response to recall antigens in the teriflunomide group did not notably differ from responses in the placebo group.

Conclusions: Following vaccination, geometric mean titers for rabies antibodies were lower with teriflunomide than with placebo. However, teriflunomide did not limit the ability to achieve seroprotective titers against this neoantigen. Evaluation of DTH showed that teriflunomide had no adverse impact on the cellular memory response to recall antigens.

Classification of evidence: This study provides Class II evidence that in normal subjects treated with teriflunomide, antibody titer responses to rabies vaccination are lower than with placebo but sufficient for seroprotection. Neurol Neuroimmunol Neuroinflamm 2015;2:e70; doi: 10.1212/ NXI.0000000000000070

\section{GLOSSARY}

$\mathbf{C I}=$ confidence interval; $\mathbf{D T H}=$ delayed-type hypersensitivity; $\mathbf{K L H}=$ keyhole limpet hemocyanin; $\mathbf{M S}=$ multiple sclerosis; PPD = tuberculin purified protein derivative; ULN = upper limit of normal; WHO = World Health Organization.

Teriflunomide is a once-daily oral immunomodulator approved for the treatment of relapsingremitting multiple sclerosis (MS). ${ }^{1,2}$ Teriflunomide blocks de novo pyrimidine synthesis through selective, noncompetitive, and reversible inhibition of dihydroorotate dehydrogenase, ${ }^{3-5}$ a mitochondrial enzyme expressed at high levels in proliferating lymphocytes. ${ }^{6}$ Through this blockade, teriflunomide exerts inhibitory effects on activated $\mathrm{T}$ and $\mathrm{B}$ cells, limiting their proliferation (without cytotoxicity) and thereby their involvement in the inflammatory processes that contribute to MS activity. ${ }^{7}$ Resting and slowly dividing lymphocytes can use salvaged pyrimidines from catabolic processes to satisfy metabolic needs and are therefore not affected by teriflunomide. ${ }^{8}$ This difference explains how teriflunomide can exert immunomodulatory

From the Montreal Neurological Institute (A.B.-O.), McGill University, Montreal, Quebec, Canada; University of Münster (H.W.), Münster, Germany; Sanofi (B.M.), Bridgewater, NJ; Genzyme, a Sanofi company (M.B., P.T., F.M.-V.), Chilly-Mazarin, France; and Fishawack Communications, Inc. (M.C.), Horsham, PA.

Go to Neurology.org/nn for full disclosures. Funding information and disclosures deemed relevant by the authors, if any, are provided at the end of the article. The Article Processing Charge was paid by Fishawack Communications, Inc., on behalf of study sponsor Genzyme, a Sanofi company. This is an open access article distributed under the terms of the Creative Commons Attribution-Noncommercial No Derivative 3.0 License, which permits downloading and sharing the work provided it is properly cited. The work cannot be changed in any way or used commercially. 
effects - by reducing proliferation of stimulated lymphocytes without obviously compromising lymphocyte homeostasis and protective immunity. ${ }^{9,10}$

Medications that alter the immune system have the potential to limit primary and secondary immune responses (e.g., the ability to mount an effective immune response to vaccinations). ${ }^{11}$ We previously investigated the effects of teriflunomide on the immune response to seasonal influenza vaccination in patients with relapsing forms of MS. ${ }^{12}$ This vaccination was considered a recall response, as most individuals had been previously in contact with the virus or had been vaccinated. This study showed that serologic responses to influenza vaccine were preserved in patients treated with teriflunomide. ${ }^{12}$

In the present study, we evaluated the antibody response to a neoantigen by using the rabies vaccination in healthy subjects treated with teriflunomide or placebo. Furthermore, we assessed delayed-type hypersensitivity (DTH) to recall antigens as a global measure of cellular memory responses.

METHODS Standard protocol approvals, registrations, and subject consents. The study was conducted in accordance with the 18th World Health Congress Recommendations, Declaration of Helsinki, and all applicable amendments. The study protocol and informed consent forms were approved by an institutional review board before initiation, and complied with local laws and regulations and applicable country guidelines. All subjects provided written informed consent.

Study design and subjects. This was a single-center, randomized, double-blind, parallel-group, placebo-controlled study conducted in Quebec, Canada. Forty-six healthy men and women aged 18-60 years were enrolled. Health status was based on a comprehensive clinical assessment, detailed medical history, and complete physical examination. Vital signs, including blood pressure and ECG readings, and laboratory parameters were required to be within normal ranges. Included subjects could not have been previously vaccinated against rabies.

Subjects had to have a positive DTH skin test to at least 1 of the 3 recall antigens tested at screening: Candida albicans, Trichophyton, or tuberculin purified protein derivative (PPD). Per protocol, any subject with a positive PPD skin test also underwent a follow-up blood interferon- $\gamma$ release assay test (QuantiFERON, QIAGEN, Valencia, CA) to rule out latent tuberculosis infection. If the QuantiFERON test was positive, the subject was excluded.

Any subject with signs of acute illness during screening was excluded. Additional criteria prohibited use of over-the-counter supplements (within 7 days of dosing) or exposure to any investigational drug (within 30 days of dosing). Women who were pregnant or breast-feeding were also excluded.

Dosing and administration. The screening period of 2-21 days was followed by a 30-day active treatment period in which subjects were randomized to teriflunomide or placebo. Subjects were randomized to treatment groups in accordance with the randomization list that was generated by the sponsor. Teriflunomide was administered as a loading dose of $70 \mathrm{mg}$ (5 14-mg tablets) once daily for 5 days (day 1-day 5) to reach steady-state therapeutic concentrations more rapidly, followed by a 14-mg dose (1 tablet) once daily for 25 days (day 6-day 30) to maintain steady-state concentrations (figure 1). Subjects randomized to placebo received an equivalent number of matching placebo tablets. Participants and study site personnel were blinded to treatment assignment.

Rabies vaccine (Imovax Rabies $1 \mathrm{~mL}$, Sanofi Pasteur, Lyon, France) was administered as an IM injection on days 5, 12, and 31 after the start of the active treatment period (i.e., the time teriflunomide steady-state plasma concentration was reached).

Antigens of $C$ albicans (1,000 units/mL, ALK Abello, Round Rock, TX), Trichophyton (1,000 units/mL, ALK Abello), and PPD (Tubersol, 5 tuberculin units per $0.1 \mathrm{~mL}$, Sanofi Pasteur, Ontario, Canada) were administered intradermally at screening (inclusion criteria) and then on treatment day 28. Skin tests were administered under a volume of $0.1 \mathrm{~mL}$, spaced at least $5 \mathrm{~cm}$ apart on the volar surface of the forearm to avoid interference.

At day 31, subjects started an 8-day drug-free period. On day 38 , upon completion of the vaccination assessment, all subjects underwent an accelerated elimination procedure for teriflunomide using orally administered cholestyramine $4 \mathrm{~g} 3$ times daily for 11 days.

Study assessments. The primary objective was to assess the antibody response to rabies vaccine in subjects treated with teriflunomide compared with subjects treated with placebo. The primary pharmacodynamic variable was the rabies-specific antibody level at each scheduled visit during the treatment period. Antibody titers to rabies vaccine were assessed using the rapid fluorescent focus inhibition test at the predose visit and at days 12, 19, 31, and 38 (performed at The Rabies Laboratory, Kansas State University, Manhattan, Kansas). The results were given in IU/mL compared with a standard reference serum. Seroprotection was defined per the World Health Organization (WHO) as a minimum antirabies antibody titer of $0.5 \mathrm{IU} / \mathrm{mL}$ for rabies vaccination. ${ }^{13}$

The effect of teriflunomide on the immune response to recall antigens was assessed as a secondary objective. A positive DTH reaction was defined as a diameter of induration $\geq 2 \mathrm{~mm}$ measured 48 hours after intradermal administration of the antigen, indicating an appropriate cellular memory response to recall antigens. ${ }^{14}$

Safety assessments included investigator- or subject-reported adverse events, clinical laboratory tests, vital signs, and physical examinations. Adverse event reports were collected throughout the study. Vital signs were performed at screening, baseline, days 12,19 , and 31 , and the end of the study. Laboratory testing, including hematology, was conducted at screening, baseline, days $5,12,19$, and 31 , and every visit until the end of the study. Plasma samples to measure teriflunomide concentrations were collected on days $6,12,19,31,38$, and 49 (end of the accelerated elimination procedure).

Subjects could withdraw at any time. Per protocol, subjects were required to discontinue if alanine aminotransferase values exceeded 3 times the upper limit of normal (ULN), if confirmed neutrophil counts were $<1,500$ cells $/ \mathrm{mm}^{3}$ with or without signs of infection, or if confirmed platelet count was $<100,000$ cells $/ \mathrm{mm}^{3}$ with or without bleeding. Serum $\alpha$-amylase or lipase values exceeding 2 times the ULN or a serum creatinine increase $>150 \mu \mathrm{mol} / \mathrm{L}$ that could not be rapidly reversed also required discontinuation.

Statistical analysis. A sample size of 20 subjects per group produces a 2-sided $90 \%$ confidence interval (CI) providing approximately $38.5 \%$ maximum imprecision for the estimation 


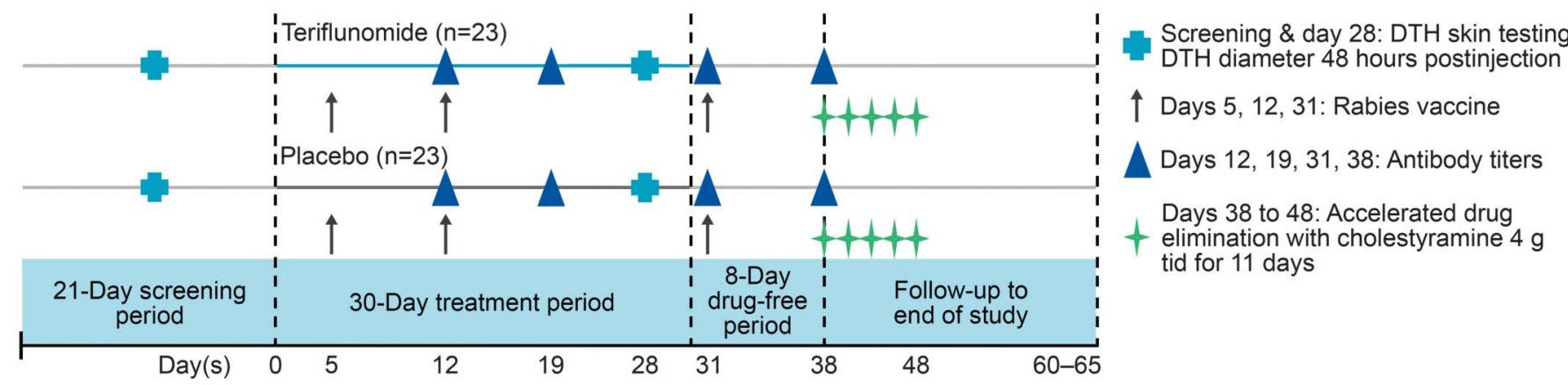

A screening period of 2-21 days was followed by an active treatment period of 30 days with teriflunomide $14 \mathrm{mg}$ or placebo. The study was complete $12-17$ days after the accelerated drug elimination phase. DTH = delayed-type hypersensitivity.

of the treatment ratio of geometric mean rabies antibody levels for subjects treated with teriflunomide vs placebo. This assumes an $\mathrm{SD}$ of 0.80 . For the primary pharmacodynamic analysis, the rabies-specific antibody levels were summarized with descriptive statistics by treatment for each scheduled time point during the treatment period. Such summaries were accompanied by a plot of geometric mean titers of rabies-specific antibody levels over time by treatment. For each scheduled visit, the ratio of geometric mean levels for subjects treated with teriflunomide vs placebo was provided along with the corresponding $90 \% \mathrm{CI}$.

The number of subjects (with percentage and by treatment group) with positive and negative DTH reactions to recall antigens after treatment was summarized for subjects with a positive DTH reaction at screening. A bar chart of the number of subjects

\section{Figure 2 Subject disposition}

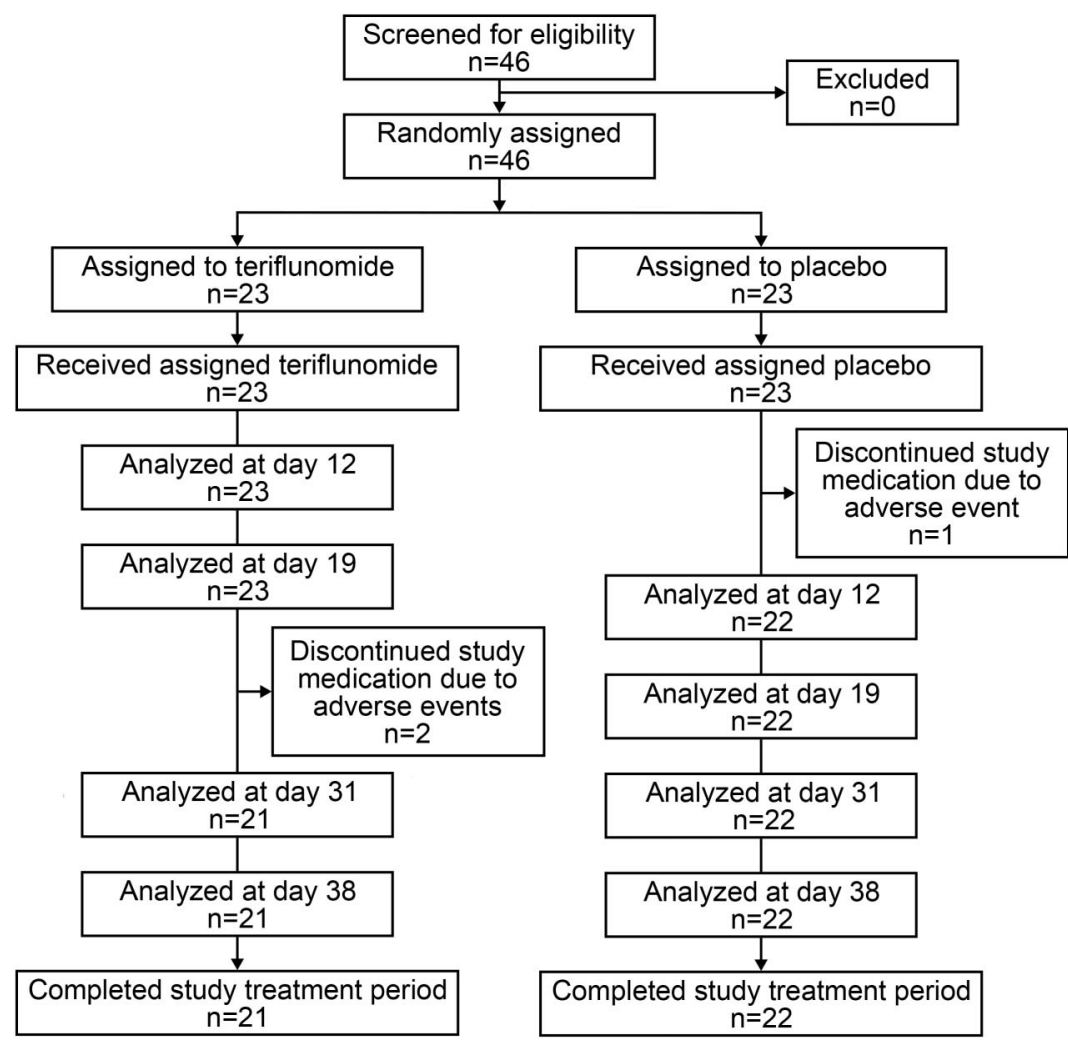

with a positive DTH reaction was provided by antigen and treatment for screening and day 30 .

Study drug levels were measured in teriflunomide-treated subjects and summarized using descriptive statistics.

All subjects exposed to study medication were included in the safety analysis. Safety was evaluated using descriptive statistics of individual clinical laboratory data, vital signs, and physical examination data. Treatment-emergent adverse events were events that occurred or worsened during the on-treatment phase (time from when the first dose was administered until the last visit, figure 1) and were assigned to the medication the subject was receiving at that time.

RESULTS Subject disposition and baseline demographics. The first subject was enrolled on August 2, 2012, and the last subject completed the study on November 28, 2012. All 46 enrolled subjects (teriflunomide, $n=23$; placebo, $n=23$ ) were randomized and treated, and all subjects completed the rabies vaccination schedule (figure 2). Two subjects in the teriflunomide group and 1 subject in the placebo group discontinued early because of treatment-emergent adverse events. Subject demographics were well-balanced (table e-1 at Neurology.org/nn). Most subjects were male (65.2\%), as commonly observed in trials including healthy volunteers; the mean age (SD) was 39.9 (11.7) years and all were white (100\%).

Pharmacokinetic evaluation. In the teriflunomide group, steady-state serum concentrations of teriflunomide were reached by day 6 and maintained through the end of treatment, with mean values ranging from 39.1 $\mu \mathrm{g} / \mathrm{mL}$ on day 12 to $43.2 \mu \mathrm{g} / \mathrm{mL}$ on day 31 . At day 38 , 8 days after stopping treatment, teriflunomide concentrations remained over $30 \mu \mathrm{g} / \mathrm{mL}$, consistent with the long half-life of the drug. Before release from the study, all subjects underwent an accelerated drug elimination procedure.

Immune response to neoantigen. Prevaccination, none of the subjects had detectable antibodies to the rabies virus. Measurable and seroprotective antibody levels (according to the WHO definition, minimum 
Table 1 Treatment geometric mean ratio of rabies antibody level with $90 \% \mathrm{Cl}$ (teriflunomide vs placebo)

$\begin{array}{lllll}\text { Scheduled day } & \begin{array}{l}\text { Teriflunomide GMT } \\ \text { (min-max), IU/mL }\end{array} & \begin{array}{l}\text { Placebo GMT (min-max), } \\ \text { IU/mL }\end{array} & \text { Treatment ratio } & 90 \% \mathrm{Cl} \\ \text { Day } 19 & 11.51(2.6-42.0) & 15.40(0.4-86.0) & 0.75 & 0.49,1.13 \\ \text { Day } 31 & 7.82(0.6-43.0) & 12.47(2.0-160.0) & 0.63 & 0.41,0.97 \\ \text { Day 38 } & 15.19(4.3-50.5) & 28.85(10.0-77.0) & 0.53 & 0.35,0.81\end{array}$

Abbreviations: $\mathrm{Cl}=$ confidence interval; GMT = geometric mean titer.

Treatment ratios are of geometric means. Day 12 (1 week after the first rabies vaccination) results are not shown because 22 of 23 subjects $(95.7 \%)$ receiving teriflunomide and 18 of 22 subjects $(81.8 \%)$ receiving placebo had antibody levels below the limit of quantification.

antirabies antibody titer) were induced following rabies vaccination in all subjects. However, geometric mean antibody titers were lower in the teriflunomide group than in the placebo group at days 19, 31, and 38 (table 1).

Seven days after the first rabies vaccination, 1 subject in the teriflunomide group and 2 subjects in the placebo group had detectable antibodies. One week after the second vaccination (day 19), all subjects had measurable antibody levels. Geometric mean antibody titers increased to $11.51 \mathrm{IU} / \mathrm{mL}$ in the teriflunomide group and $15.4 \mathrm{IU} / \mathrm{mL}$ in the placebo group. Two weeks after the second rabies vaccination (day 31), geometric mean antibody titers decreased to $7.82 \mathrm{IU} / \mathrm{mL}$ in the teriflunomide group and 12.47 $\mathrm{IU} / \mathrm{mL}$ in the placebo group, with a significant between-group difference based on the 90\% CI. However, at this time point all subjects had achieved seroprotection, as defined by the $0.5 \mathrm{IU} / \mathrm{mL}$

Figure 3 Immune response to neoantigen: Geometric mean titers of antirabies antibody levels over time

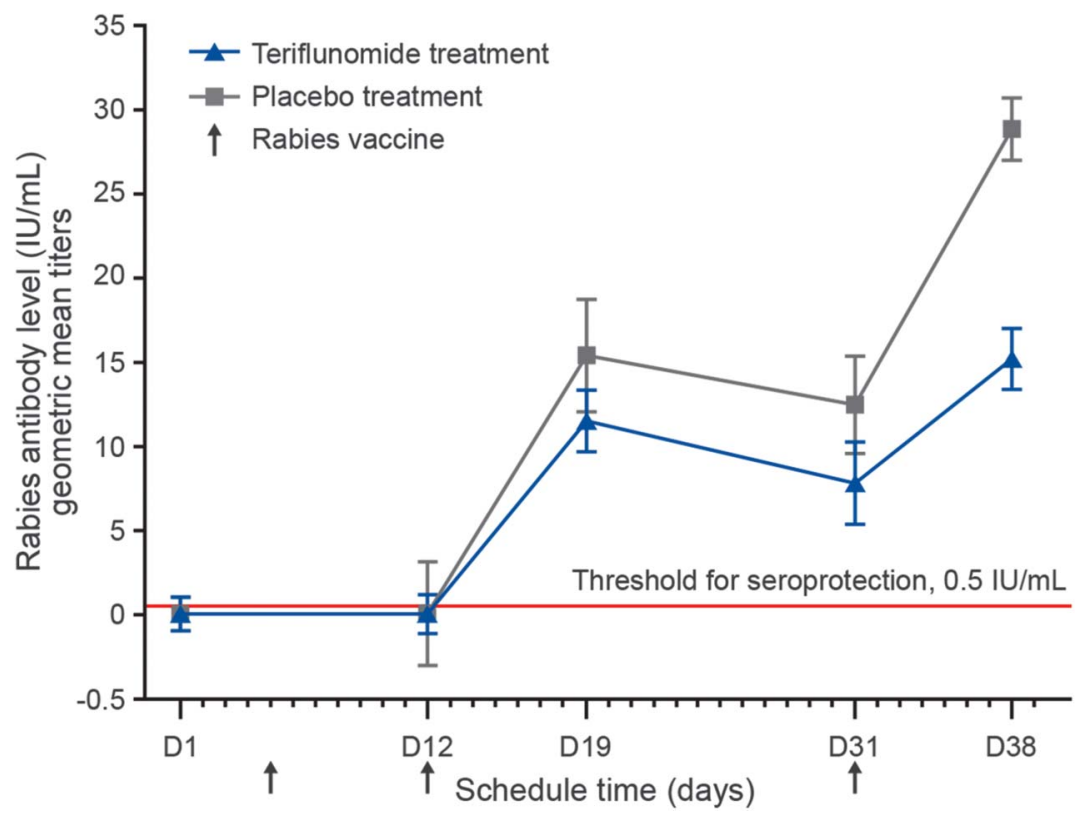

At days 31 and 38 , all subjects achieved antibody titers above the $0.5 \mathrm{IU} / \mathrm{mL}$ threshold for seroprotection. Error bars show SD for each data point. threshold (titers ranged from $0.6 \mathrm{IU} / \mathrm{mL}$ to 43.0 $\mathrm{IU} / \mathrm{mL}$ in the teriflunomide group and from 2.0 $\mathrm{IU} / \mathrm{mL}$ to $160.0 \mathrm{IU} / \mathrm{mL}$ in the placebo group; figure 3 and tables 1 and 2).

Following the cessation of active treatment and 1 week after the third rabies vaccination (day 38), geometric mean antibody titers increased but were still significantly lower (based on the 90\% CI) in the teriflunomide group $(15.19 \mathrm{IU} / \mathrm{mL})$ than in the placebo group $(28.85 \mathrm{IU} / \mathrm{mL})$. However, all titers remained well above the $0.5 \mathrm{IU} / \mathrm{mL}$ threshold for seroprotection, ranging from $4.3 \mathrm{IU} / \mathrm{mL}$ to $50.5 \mathrm{IU} / \mathrm{mL}$ in the teriflunomide group and from $10.0 \mathrm{IU} / \mathrm{mL}$ to $77.0 \mathrm{IU} / \mathrm{mL}$ in the placebo group (figure 3 and tables 1 and 2).

Delayed-type hypersensitivity to recall antigens. The number of subjects with positive DTH at screening and at day 30 is displayed in figure 4 . By the end of treatment, the number of subjects with a positive DTH response to $C$ albicans or Trichophyton was slightly lower than at screening in both the teriflunomide and placebo groups. The number of subjects with a positive DTH to PPD at screening was low and was unchanged with teriflunomide or placebo treatment. Overall, the responses to recall antigens in the teriflunomide group, both before and at the end of treatment, did not differ notably from responses in the placebo group (figure 4), an outcome supported by descriptive statistics.

Safety and tolerability. There was a low incidence of treatment-emergent adverse events in the teriflunomide $(17.4 \%)$ and placebo $(30.4 \%)$ groups (table e-2). All events were mild to moderate in intensity, and no serious adverse events were reported.

Three subjects had lipase increase $>2$ times the ULN ( 2 subjects in the teriflunomide group and 1 subject in the placebo group). No cases of lipase increase in either group were associated with other laboratory abnormalities, and values normalized within 5 days. All occurrences were asymptomatic and pancreatic ultrasound showed no abnormalities. Per protocol, treatment was discontinued for any subject with lipase increase $>2$ times the ULN. One subject receiving teriflunomide with lipase increase of 4.4 times the 
Table 2 Proportion of subjects achieving seroprotection (antibody titers of $\geq 0.5 \mathrm{IU} / \mathrm{mL}$ )

\begin{tabular}{llll} 
& \multicolumn{2}{c}{ Seroprotection, $\mathrm{n} / \mathrm{N}(\%)$} & \multicolumn{1}{c}{$\begin{array}{l}\text { Difference }(\mathbf{9 0 \%} \mathrm{Cl}), \\
\text { teriflunomide vs placebo }\end{array}$} \\
\cline { 2 - 4 } Scheduled day & Teriflunomide $(\mathrm{N}=23)$ & Placebo $(\mathbf{N}=22)$ & $-0.09(-0.24,0.03)$ \\
Day 12 & $0 / 23(0)$ & $2 / 22(9.1)$ & $0.05(-0.07,0.18)$ \\
Day 19 & $23 / 23(100)$ & $21 / 22(95.5)$ & $\mathrm{NA}$ \\
Day 31 & $21 / 21(100)$ & $22 / 22(100)$ & NA
\end{tabular}

Abbreviations: $\mathrm{Cl}=$ confidence interval; $\mathrm{NA}=$ not applicable (no difference $[90 \% \mathrm{Cl}]$ reported).

Two subjects in the teriflunomide group discontinued early and are therefore not included in day 31 and day 38 summaries. One subject in the placebo group is not included postbaseline due to discontinuation of the study drug after 7 days of treatment. At days 31 and 38, all subjects achieved antibody titers above the $0.5 \mathrm{IU} / \mathrm{mL}$ threshold for seroprotection.

ULN at day 19 and 1 subject receiving placebo with lipase increase of 2.6 times the ULN at day 7 were therefore discontinued. The additional subject with lipase increase in the teriflunomide group had levels increase to 2.2 times the ULN at day 31, 1 day after the last dose was administered.

Low neutrophil values (grade 2 or greater: $<1,500$ cells $/ \mathrm{mm}^{3}$ ) were reported for 5 subjects receiving teriflunomide. For 4 of these subjects, values normalized within a few days while they remained on treatment. One subject had a subsequent neutrophil value of 980 cells $/ \mathrm{mm}^{3}$ (grade 3) at day 25 and was withdrawn from treatment per protocol requirements. There were no infections reported for any subject with abnormal neutrophil counts. Laboratory analysis also showed that mean changes from baseline in neutrophil and lymphocyte counts were small in magnitude (figures e-1a and e-1b).

No safety concerns were associated with rabies vaccination. Only 2 occurrences of local pain or erythema at the injection site were reported in the placebo group.

DISCUSSION Our results demonstrate that healthy subjects exposed for 1 month to a therapeutic level of teriflunomide were able to mount seroprotective immune responses against the rabies vaccine, used

Figure 4 Number of subjects with positive DTH response to recall antigens before and at the end of treatment

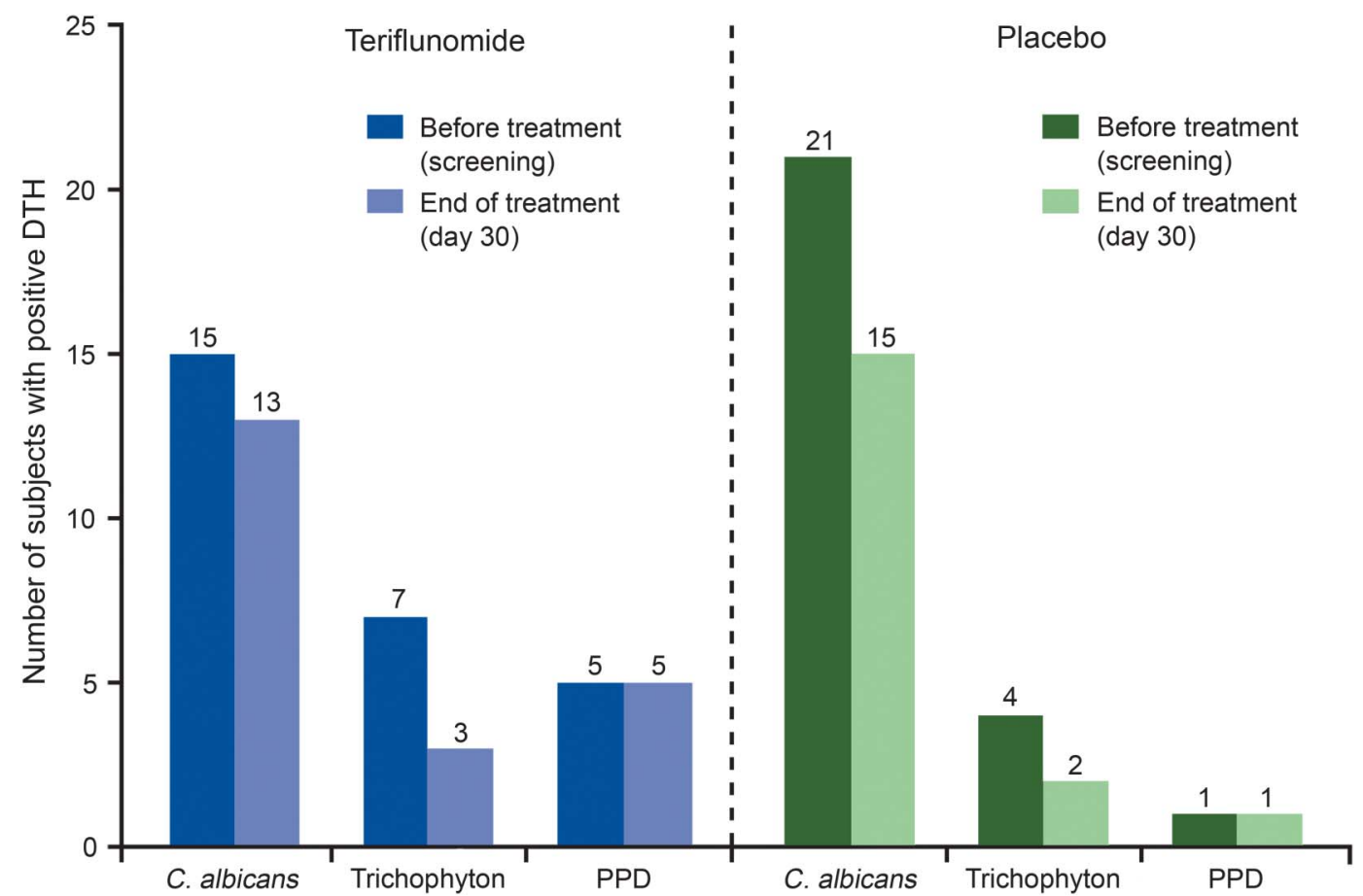

A positive delayed-type hypersensitivity (DTH) reaction was defined as a diameter of induration $\geq 2 \mathrm{~mm}$ measured 48 hours after intradermal administration of the antigen. Three subjects ( 2 receiving teriflunomide, 1 receiving placebo) had positive DTH for at least one antigen at baseline but discontinued prematurely from the study and did not have DTH testing at the end of treatment. These subjects are not included. . albicans $=$ Candida albicans; PPD = tuberculin purified protein derivatives. 
as a neoantigen. Although antibody levels for rabies in general were lower over time in the teriflunomide group than in the placebo group, all subjects achieved titers well above the $0.5 \mathrm{IU} / \mathrm{mL}$ threshold, defined by the WHO as appropriate seroprotection following rabies vaccination. ${ }^{13}$

Several neoantigens, such as the Phi-X-174, a viruslike bacteriophage, or the keyhole limpet hemocyanin $(\mathrm{KLH})$ protein, have been used in either healthy subjects or patients to investigate the impact of a disease-modifying therapy (efalizumab, ${ }^{14}$ fingolimod,,${ }^{15}$ rituximab or methotrexate, ${ }^{16}$ or natalizumab ${ }^{17}$ ) on the immune response. We considered the rabies vaccine a more practical neoantigen for our study because it has a well-defined vaccination schedule, does not contain live virus, requires no extemporaneous preparation to use, and is well-tolerated, even in patients with humoral or cellular deficiency. ${ }^{18-20}$ Previous studies have also found the rabies vaccine to be a useful tool to investigate the immune response in healthy subjects or patients. ${ }^{20-23}$ Unlike the Phi-X-174 or KLH neoantigens, the rabies vaccine provides a threshold reference for seroprotection (antibody titer of $0.5 \mathrm{IU} / \mathrm{mL}^{13}$ ), allowing for a biologically relevant evaluation of the effect of teriflunomide on the functional immune response.

In addition, we assessed in parallel the occurrence of DTH reactions - a global measure of cellular memory responses - before and at the end of treatment. We found that teriflunomide had no deleterious impact on the cellular responses to a sample of recall antigens ( $C$ albicans, Trichophyton, PPD). These findings supplement those of a previous phase 2 trial (the TERIVA study; NCT01403376) in which patients with relapsing MS receiving teriflunomide mounted effective humoral antibody responses to seasonal influenza vaccination. ${ }^{12}$ Safety data from teriflunomide clinical studies also support this view, since general immune competence did not seem to be significantly altered in phase 3 double-blind treatment periods (as measured by mild decreases in neutrophil and lymphocyte counts that remained within normal range with no associated infection $)^{24}$ and over long-term extensions with up to 12 years of continuous exposure. ${ }^{25,26}$ In addition, several preclinical studies have demonstrated that teriflunomide does not exert cytotoxic effects and preferentially inhibits proliferative activity of strongly stimulated adaptive immune cells, providing further support for the selective impact of this agent on immune responses. ${ }^{9,27}$

One potential limitation of our study is the relatively short exposure period (30 days), raising the question of generalizability of our findings to patients with longer-term exposure to teriflunomide in the clinical setting. By using a loading dose, our regimen achieved steady-state teriflunomide plasma concentrations after
5 days, which are comparable to those documented with longer-term teriflunomide 14-mg dosing in patients with MS. ${ }^{28}$ Teriflunomide levels remained stable in our teriflunomide-treated cohort throughout the 25 days of 14-mg dosing, during which time we also observed a mild decrease in neutrophil and lymphocyte counts, similar in magnitude to the mild decreases observed in the clinical setting in patients with MS treated with teriflunomide. ${ }^{24}$ Together, these pharmacokinetic and pharmacodynamic observations provide some reassurance regarding the generalizability of our findings. Also noteworthy are the previously described clinical observations, ${ }^{25,26}$ including safety profiles related to immune competence, which support the view that longer-term administration of teriflunomide had no cumulative immune suppressive effect. While an immune response to rabies neoantigen does not necessarily indicate response to other antigens, our results are further supported by the TERIVA study results, in which patients continuously treated with teriflunomide for more than 2 years were able to mount effective responses toward multiple influenza vaccine strains. ${ }^{12}$ To ensure our results are not interpreted beyond the context of these studies, we refer to the teriflunomide prescribing information, ${ }^{1,2}$ which recommends avoiding live vaccines, and note that although no general concern has been identified, vaccination success might be quantitatively and qualitatively affected by teriflunomide.

Our current study was performed in healthy subjects, not in patients with MS. Based on a lack of information on responses to rabies vaccination in patients with MS and the lack of a clear protective benefit from a rarely encountered infection, we felt that a study in healthy volunteers was more appropriate and the most practical and acceptable option to assess immune responses to rabies vaccination in humans. Nevertheless, patients with MS have been described as having responses to vaccinations that are similar to those observed in healthy subjects, with no obvious deficits in immune responses. ${ }^{29}$ Therefore, our findings are likely to remain relevant to the MS population.

Since infections may increase the frequency of MS activity $^{30}$ and MS relapses associated with infections have been related to more severe long-term neurologic sequelae, ${ }^{31}$ establishing safe and effective vaccination practices for patients receiving immune-targeted MS therapies is important. In our cohort of teriflunomidetreated individuals, the rabies vaccination was well-tolerated, with a safety profile consistent with previous studies. ${ }^{24,25}$ Our findings, supplemented by the TERIVA study, ${ }^{12}$ thus support the view that vaccinations with either inactivated neoantigen or recall antigens are effective while taking teriflunomide and that treatment with teriflunomide need not be interrupted if vaccination is deemed appropriate. 
Our findings indicate that teriflunomide does not limit the ability to achieve adequate seroprotection against neoantigen, an indicator of the capacity to mount primary adaptive humoral immune responses, as all subjects achieved titers above the $0.5 \mathrm{IU} / \mathrm{mL}$ threshold following rabies vaccination. Furthermore, teriflunomide did not interfere with the cellular response to recall antigens, as demonstrated using DTH with 3 different recall antigens over a 1-month observation period. These results are consistent with the emerging view that teriflunomide selectively modulates immune responses, thereby limiting auto-aggressive immune responses involved in MS disease activity without functionally compromising a broad range of host protective immune responses.

\section{AUTHOR CONTRIBUTIONS}

Amit Bar-Or, Barry Miller, Myriam Benamor, Philippe Truffinet, and Françoise Menguy-Vacheron contributed to the development of the study design, data analysis, and interpretation. Heinz Wiendl was involved in the data analysis and interpretation. They were all involved in the development, reviewing, and revising of the manuscript. Editorial assistance in preparing the manuscript (funded by Genzyme, a Sanofi company) was provided by Meg Church, who assisted authors in the preparation and editing of the manuscript text, figures, and tables; addressed author, reviewer, and editorial comments; and assisted with the submission process.

\section{ACKNOWLEDGMENT}

The manuscript was reviewed by Thierry Aupérin, PhD, of Genzyme, a Sanofi company.

\section{STUDY FUNDING}

This study was funded by Genzyme, a Sanofi company. Editorial support was funded by Genzyme.

\section{DISCLOSURE}

A. Bar-Or is on the scientific advisory board for Diogenix, Ono Pharmacia, Receptos, Roche, Novartis, GSK, and Guthy Jackson Greater Good Foundation; is on the editorial board for Neurology and Clinical and Experimental Neuroimmunology; has consulted for Diogenix, Ono Pharmacia, Receptos, Roche, Novartis, and GSK; and has received research support from Novartis, EMD Serono, and Genzyme-Sanofi. H. Wiendl has received honoraria/travel expenses from Bayer, Biogen Idec/Elan, Sanofi-Aventis, Schering, Serono, and Teva; has been a consultant for Serono, Medac, Sanofi-Aventis/TEVA, Biogen Idec, BayerVita/Schering, Novartis, and NovoNordisk; and has received research support from Bayer, Biogen Idec/Elan, Sanofi-Aventis, Schering GmbH, Merck Serono, Teva, Novartis, Medac, Genzyme, and NovoNordisk. B. Miller is employed by Sanofi. M. Benamor is employed by Genzyme, a Sanofi company. P. Truffinet is employed by Genzyme/Sanofi and receives stocks and salary as an employee. M. Church is a former employee of Fishawack Communications and was contracted to provide editorial services to Sanofi, and was funded by Genzyme, a Sanofi company. F. Menguy-Vacheron is a former employee of Genzyme, a Sanofi company. Go to Neurology.org/nn for full disclosures.

Received September 8, 2014. Accepted in final form December 22, 2014.

\section{REFERENCES}

1. AUBAGIO Prescribing Information. Cambridge, MA: Genzyme Corporation, a Sanofi company; 2012.

2. AUBAGIO Summary of Product Characteristics. Paris, France: sanofi-aventis groupe; 2013.
3. Bruneau JM, Yea CM, Spinella-Jaegle S, et al. Purification of human dihydro-orotate dehydrogenase and its inhibition by A77 1726, the active metabolite of leflunomide. Biochem J 1998;336:299-303.

4. Cherwinski HM, Cohn RG, Cheung P, et al. The immunosuppressant leflunomide inhibits lymphocyte proliferation by inhibiting pyrimidine biosynthesis. J Pharmacol Exp Ther 1995;275:1043-1049.

5. Ruckemann K, Fairbanks LD, Carrey EA, et al. Leflunomide inhibits pyrimidine de novo synthesis in mitogenstimulated T-lymphocytes from healthy humans. J Biol Chem 1998;273:21682-21691.

6. Loffler M, Klein A, Hayek-Ouassini M, Knecht W, Konrad L. Dihydroorotate dehydrogenase mRNA and protein expression analysis in normal and drug-resistant cells. Nucleosides Nucleotides Nucleic Acids 2004;23: 1281-1285.

7. Gold R, Wolinsky JS. Pathophysiology of multiple sclerosis and the place of teriflunomide. Acta Neurol Scand 2011;124:75-84.

8. Claussen MC, Korn T. Immune mechanisms of new therapeutic strategies in MS: teriflunomide. Clin Immunol 2012;142:49-56.

9. Li L, Liu J, Delohery T, Zhang D, Arendt C, Jones C. The effects of teriflunomide on lymphocyte subpopulations in human peripheral blood mononuclear cells in vitro. J Neuroimmunol 2013;265:82-90.

10. Bar-Or A, Pachner A, Menguy-Vacheron F, Kaplan J, Wiendl $\mathrm{H}$. Teriflunomide and its mechanism of action in multiple sclerosis. Drugs 2014;74:659-674.

11. Loebermann M, Winkelmann A, Hartung HP, Hengel H, Reisinger EC, Zettl UK. Vaccination against infection in patients with multiple sclerosis. Nat Rev Neurol 2012;8: 143-151.

12. Bar-Or A, Freedman M, Kremenchutzky M, et al. Teriflunomide effect on immune response to influenza vaccine in patients with multiple sclerosis. Neurology 2013;81:552-558.

13. World Health Organization. Updated WHO Position Paper on Rabies Vaccines. Available at: http://www.who. int/immunization/Rabies_slides_Aug_2010.pdf. Accessed November 24, 2014.

14. Krueger JG, Ochs HD, Patel P, Gilkerson E, GuttmanYassky E, Dummer W. Effect of therapeutic integrin (CD11a) blockade with efalizumab on immune responses to model antigens in humans: results of a randomized, single blind study. J Invest Dermatol 2008;128:2615-2624.

15. Boulton C, Meiser K, David OJ, Schmouder R. Pharmacodynamic effects of steady-state fingolimod on antibody response in healthy volunteers: a 4-week, randomized, placebo-controlled, parallel-group, multiple-dose study. J Clin Pharmacol 2012;52:1879-1890.

16. Bingham CO III, Looney RJ, Deodhar A, et al. Immunization responses in rheumatoid arthritis patients treated with rituximab: results from a controlled clinical trial. Arthritis Rheum 2010;62:64-74.

17. National Institutes of Health. The effects of Tysabri treatment on vaccination response and lymphocyte subsets in subjects with relapsing forms of multiple sclerosis. Available at: http://clinicaltrials.gov/ct2/show/NCT00536120. Accessed November 25, 2014.

18. Cramer CH II, Shieck V, Thomas SE, Kershaw DB, Magee JC, Lopez MJ. Immune response to rabies vaccination in pediatric transplant patients. Pediatr Transplant 2008;12:874-877. 
19. Thisyakorn U, Pancharoen C, Ruxrungtham K, et al. Safety and immunogenicity of preexposure rabies vaccination in children infected with human immunodeficiency virus type 1 . Clin Infect Dis 2000;30:218.

20. Brinkman DM, Jol-van der Zijde CM, ten Dam MM, et al. Vaccination with rabies to study the humoral and cellular immune response to a T-cell dependent neoantigen in man. J Clin Immunol 2003;23: 528-538.

21. Azzoni L, Foulkes AS, Firnhaber C, et al. ART therapy interruptions result in loss of protective humoral immunity to neoantigens in $\mathrm{HIV}$-infected individuals. AIDS 2012;26:1355-1362.

22. Brinkman DM, Jol-van der Zijde CM, ten Dam MM, et al. Resetting the adaptive immune system after autologous stem cell transplantation: lessons from responses to vaccines. J Clin Immunol 2007;27:647-658.

23. Gelinck LB, Jol-van der Zijde CM, JansenHoogendijk AM, et al. Restoration of the antibody response upon rabies vaccination in HIV-infected patients treated with HAART. AIDS 2009;23:2451-2458.

24. O'Connor P, Wolinsky JS, Confavreux C, et al. Randomized trial of oral teriflunomide for relapsing multiple sclerosis. N Engl J Med 2011;365:1293-1303.
25. Freedman M, Wolinsky JS, Comi G, et al. Long-term safety and efficacy of teriflunomide in patients with relapsing forms of multiple sclerosis in the TEMSO extension trial. Mult Scler J 2013;19(suppl 11):P544.

26. Singer B, Comi G, Miller A, Freedman M, Benamor M, Truffinet P. Teriflunomide treatment is not associated with increased risk of infections: pooled data from the teriflunomide development program. Neurology 2014;82(suppl 10):P2.194.

27. Posevitz V, Chudyka D, Schneider-Hohendorf T, Schwab N, Kurth F, Wiendl H. Teriflunomide selectively suppresses antigen induced T-cell expansion in a TCR avidity dependent fashion. Mult Scler J 2012;18:P1107.

28. Oh J, O'Connor PW. An update of teriflunomide for treatment of multiple sclerosis. Ther Clin Risk Manag 2013;9:177-190.

29. Moriabadi NF, Niewiesk S, Kruse N, et al. Influenza vaccination in MS: absence of T-cell response against white matter proteins. Neurology 2001;56:938-943.

30. De Keyser J, Zwanikken C, Boon M. Effects of influenza vaccination and influenza illness on exacerbations in multiple sclerosis. J Neurol Sci 1998;159:51-53.

31. Buljevac D, Flach HZ, Hop WC, et al. Prospective study on the relationship between infections and multiple sclerosis exacerbations. Brain 2002;125:952-960. 


\section{Neurology \\ Neuroimmunology \& Neuroinflammation}

\section{Randomized study of teriflunomide effects on immune responses to neoantigen and recall antigens}

Amit Bar-Or, Heinz Wiendl, Barry Miller, et al.

Neurol Neuroimmunol Neuroinflamm 2015;2;

DOI 10.1212/NXI.0000000000000070

\section{This information is current as of February 12, 2015}

Updated Information \&
Services
Supplementary Material
References
Citations
Subspecialty Collections
Permissions \& Licensing
Reprints

Updated Information \& including high resolution figures, can be found at:

http://nn.neurology.org/content/2/2/e70.full.html

Supplementary material can be found at:

http://nn.neurology.org/content/suppl/2015/02/12/2.2.e70.DC1

This article cites 27 articles, 3 of which you can access for free at: http://nn.neurology.org/content/2/2/e70.full.html\#\#ref-list-1

This article has been cited by 2 HighWire-hosted articles: http://nn.neurology.org/content/2/2/e70.full.html\#\#otherarticles

This article, along with others on similar topics, appears in the following collection(s):

Multiple sclerosis

http://nn.neurology.org//cgi/collection/multiple_sclerosis

Information about reproducing this article in parts (figures,tables) or in its entirety can be found online at:

http://nn.neurology.org/misc/about.xhtml\#permissions

Information about ordering reprints can be found online:

http://nn.neurology.org/misc/addir.xhtml\#reprintsus

Neurol Neuroimmunol Neuroinflamm is an official journal of the American Academy of Neurology.

Published since April 2014, it is an open-access, online-only, continuous publication journal. Copyright $\odot$ 2015 American Academy of Neurology. All rights reserved. Online ISSN: 2332-7812.

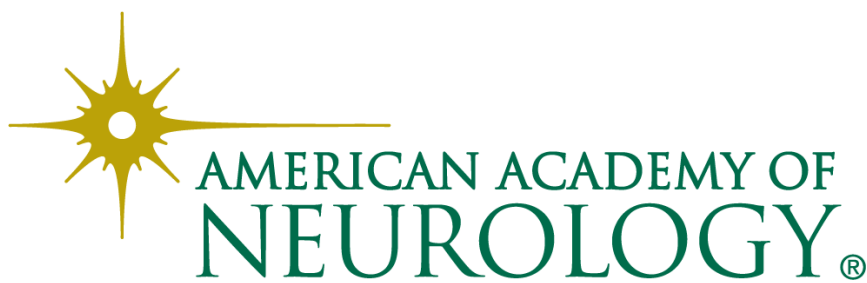

